\title{
Article \\ Confocal Fluorescence Microscopy and Confocal Raman Microspectroscopy of X-ray Irradiated LiF Crystals
}

\author{
Francesca Bonfigli ${ }^{1} * *$, Sabina Botti ${ }^{1}$, , Angelica Cecilia ${ }^{2}$, Rosa Maria Montereali ${ }^{1}{ }^{\oplus}$, Enrico Nichelatti ${ }^{3}$, \\ Valentina Nigro ${ }^{1} \mathbb{D}$, Massimo Piccinini ${ }^{1}$ and Maria Aurora Vincenti ${ }^{1}$
}

1 Photonics Micro- and Nano-Structures Laboratory, Fusion and Technologies for Nuclear Safety and Security Department, ENEA C.R. Frascati, V. E. Fermi, 45, 00044 Frascati, Italy; sabina.botti@enea.it (S.B.); rosa.montereali@enea.it (R.M.M.); valentina.nigro@enea.it (V.N.); massimo.piccinini@enea.it (M.P.); aurora.vincenti@enea.it (M.A.V.)

2 Institute for Photon Science and Synchrotron Radiation, Karlsruhe Institute of Technology (KIT), D-76344 Karlsruhe, Germany; angelica.cecilia@kit.edu

3 Photonics Micro- and Nano-Structures Laboratory, Fusion and Technologies for Nuclear Safety and Security Department, ENEA C.R. Casaccia, V. Anguillarese 301, S. Maria di Galeria, 00123 Rome, Italy; enrico.nichelatti@enea.it

* Correspondence: francesca.bonfigli@enea.it

Citation: Bonfigli, F.; Botti, S.; Cecilia, A.; Montereali, R.M.; Nichelatti, E.; Nigro, V.; Piccinini, M.; Vincenti, M.A. Confocal Fluorescence Microscopy and Confocal Raman Microspectroscopy of X-ray Irradiated LiF Crystals. Condens. Matter 2021, 6, 37. https://doi.org/ $10.3390 /$ condmat 6040037

Academic Editor: Alessandro Scordo

Received: 30 September 2021

Accepted: 15 October 2021

Published: 20 October 2021

Publisher's Note: MDPI stays neutral with regard to jurisdictional claims in published maps and institutional affiliations.

Copyright: (C) 2021 by the authors Licensee MDPI, Basel, Switzerland. This article is an open access article distributed under the terms and conditions of the Creative Commons Attribution (CC BY) license (https:// creativecommons.org/licenses/by/ $4.0 /)$.

\begin{abstract}
Radiation-induced color centers locally produced in lithium fluoride ( $\mathrm{LiF})$ are successfully used for radiation detectors. LiF detectors for extreme ultraviolet radiation, soft and hard X-rays, based on photoluminescence from aggregate electronic defects, are currently under development for imaging applications with laboratory radiation sources, as well as large-scale facilities. Among the peculiarities of LiF-based detectors, noteworthy ones are their very high intrinsic spatial resolution across a large field of view, wide dynamic range, and versatility. LiF crystals irradiated with a monochromatic $8 \mathrm{keV}$ X-ray beam at KIT synchrotron light source (Karlsruhe, Germany) and with the broadband white beam spectrum of the synchrotron bending magnet have been investigated by optical spectroscopy, laser scanning confocal microscopy in fluorescence mode, and confocal Raman micro-spectroscopy. The 3D reconstruction of the distributions of the color centers induced by the X-rays has been performed with both confocal techniques. The combination of the LiF crystal capability to register volumetric $X$-ray mapping with the optical sectioning operations of the confocal techniques has allowed performing 3D reconstructions of the $X$-ray colored volumes and it could provide advanced tools for 3D X-ray detection.
\end{abstract}

Keywords: radiation detectors; color centers; lithium fluoride; confocal fluorescence microscopy; Raman confocal micro-spectroscopy

\section{Introduction}

Lithium fluoride $(\mathrm{LiF})$ is a radiation-sensitive material in which ionizing radiations, such as charged particles (ions and electrons) and photons (EUV, X-rays, and $\gamma$-rays), can efficiently generate primary and aggregate color centers (CCs) that are stable at room temperature. The $2 \mathrm{D}$ imaging $\mathrm{LiF}$ detectors rely on the photoluminescence of radiationinduced electronic defects, known as CCs, in particular $\mathrm{F}_{2}$ and $\mathrm{F}_{3}{ }^{+}$defects, which consist of two electrons bound to two and three close anion vacancies, respectively. These defects have almost overlapping broad absorption bands ( $\mathrm{M}$ band) peaked at about $450 \mathrm{~nm}$ [1] and therefore, they can be simultaneously excited with a single pump wavelength in the blue spectral interval. On the other hand, they exhibit two different Stokes-shifted broad emission bands in the green $\left(\mathrm{F}_{3}{ }^{+}\right)$and red $\left(\mathrm{F}_{2}\right)$ spectral ranges [1]. Several peculiar features of such solid-state $\mathrm{LiF}$ luminescent detectors, i.e., very high spatial resolution [2,3] over a large field of view, wide dynamic range [4], versatility and simplicity of use, and sensitivity of the optical fluorescence reading technique, make them very promising and attractive for 
X-ray imaging applications [5]. LiF-based imaging detectors have been proposed and tested by using several X-ray sources, such as laboratory sources [3,5,6] and large-scale facilities [7], for soft X-ray microscopy of biological specimens [8], phase contrast imaging [9], and for X-ray beam characterizations [5]. LiF crystals and films were used as imaging detectors for 2D visualization of pulsed soft [10] and hard [4] X-ray FEL beam. Moreover, volumetric detection for 3D X-ray imaging applications by using radiation-induced defects in several insulating materials, such as LiF crystal [11] and phosphate glass [12] at different X-ray energies and sources, is a topical task nowadays. Investigations of luminescent materials for 3D dose mapping and imaging of ionizing radiations are crucial for dosimetry and optical data storage applications. In this paper, $\mathrm{LiF}$ crystals irradiated with $8 \mathrm{keV}$ monochromatic $\mathrm{X}$-rays and with the broadband white beam spectrum of a synchrotron bending magnet at the synchrotron light source of the Karlsruhe Institute of Technology (KIT, Germany) have been investigated with confocal optical systems: fluorescence microscopy and Raman micro-spectroscopy. The 3D reconstruction of the X-ray-induced CC distributions has been performed with both confocal techniques, obtaining fluorescence and Raman mapping of X-ray colored volumes.

\section{Materials and Methods}

Commercial LiF crystals in the form of squared plates polished on both surfaces were irradiated at the synchrotron light source of KIT. A LiF crystal $\left(5 \times 5 \mathrm{~mm}^{2}, 0.5 \mathrm{~mm}\right.$ thickness, in the following referred to as LiFC1) was irradiated with the broadband white beam spectrum of a synchrotron bending magnet and a LiF crystal $\left(10 \times 10 \mathrm{~mm}^{2}, 1 \mathrm{~mm}\right.$ thickness, in the following referred to as LiFC2) was irradiated with the $8 \mathrm{keV}$ monochromatic X-ray beam. The crystals were placed on axis of the synchrotron beam with the possibility of movement in the $X$ and $Y$ directions perpendicularly to the beam axis, and they were irradiated at normal angle of incidence. The LiFC1 sample was irradiated with the broadband white beam (3-40 keV) with a flux of $10^{16} \mathrm{ph} / \mathrm{s}$. When the beamline works in white-beam mode, the only optical component that is positioned in the beam path is a $500-\mu \mathrm{m}$ thick polished beryllium window. The attenuation exerted by the Be-window as well as the contribution of the air gap between the shutter and the sample were considered to calculate the photon flux impinging on the LiFC1 sample, which was estimated to be around $2 \times 10^{13} \mathrm{ph} /\left(\mathrm{s} \cdot \mathrm{mm}^{2}\right)$. In the irradiation of the LiFC1 sample, a commercial test pattern X500-200-30 (Xradia, Pleasanton, CA, USA), consisting of 330-nm thick gold mask deposited on a $(500 \times 500) \mu \mathrm{m}^{2} \mathrm{Si}_{3} \mathrm{~N}_{4}$ window, was positioned at a distance of $26 \mathrm{~cm}$ from the exit of the beam (at $29 \mathrm{~m}$ from the irradiation source). The propagation distance between the test pattern and the $\mathrm{LiFC} 2$ sample was set equal to $17.5 \mathrm{~cm}$, thus performing a lensless projection imaging experiment $[13,14]$. To impress the $\mathrm{X}$-ray image of the test pattern on the LiFC1 detector, the test pattern was irradiated with several exposure times between $1 \mathrm{~s}$ and $60 \mathrm{~s}$. The beam size was set equal to $(800 \times 800) \mu \mathrm{m}^{2}$. The LiFC2 sample was also irradiated by the monochromatic $8 \mathrm{keV} \mathrm{X-ray} \mathrm{beam} \mathrm{for} 112 \mathrm{~s}$ on a uniform area of $4 \times 4 \mathrm{~mm}^{2}$, with an estimated dose of about $6 \mathrm{~Gy} / \mathrm{s}$.

The Photoluminescence (PL) and Photoluminescence Excitation (PLE) spectra of the $\mathrm{X}$-ray irradiated LiF crystals were collected at room temperature (RT) with a Horiba Scientific Fluorolog-3 spectrofluorimeter Model FL3-11, equipped with a 450 W Xenon lamp, automatic slits, single-grating excitation, emission spectrometers, and a photomultiplier for PL signal detection, adopting a front-face detecting geometry. The optical absorption measurements of the irradiated LiF crystals were performed by using a Perkin-Elmer Lambda 1050 spectrophotometer at normal incidence. The spectral range was set to 190-1000 nm with 1-nm resolution.

Both irradiated $\mathrm{LiF}$ plates store volumetric information through local generation of stable CCs produced by X-rays along the crystal depth. After irradiation, LiF crystals (LiFC1 and LiFC2) were analyzed by a confocal laser scanning microscope (CLSM) operating in fluorescence mode and by a confocal laser scanning Raman micro-spectrometer. In the case of the CLSM reading system (Nikon $80 \mathrm{i}-\mathrm{C} 1$ ), the LiF crystals were illuminated by 
a 445-nm continuous laser (with nominal output power of $1.5 \mathrm{~mW}$ ) pumping in the CC absorption band peaked at about $450 \mathrm{~nm}$. The spectrally-integrated PL signal was detected by photomultipliers. The CLSM is a significant evolution of the conventional optical microscope. By scanning along the $\mathrm{Z}$ optical axis, the CLSM performs an optical sectioning of the observed sample by using a pinhole in front of the detector positioned in a conjugate plane with respect to the focus one and detecting only signals from the in-focus plane by eliminating signals from out-of-focus ones. The optical sectioning allows obtaining a tridimensional (3D) reconstruction of the sample [13]. In previous works, the CLSM system in fluorescence mode was used as reading system to detect 2D high-spatial-resolution and high-contrast X-ray images stored in irradiated LiF crystal detectors $[7,14]$. In this work, the optical sectioning capabilities have been exploited to reconstruct the 3D depth distribution of the CCs in the colored volumes. The 2D and 3D Raman maps were measured by a Raman spectrometer combined with a confocal microscope (Horiba XploRA Plus) by exciting with a 532-nm wavelength laser. When a Raman spectrometer is combined with a confocal optical microscope, it is possible to acquire the information of a complete Raman spectrum in each point of the 2D and 3D maps by scanning the sample in XYZ directions.

\section{Results and Discussion}

Figure 1a shows the optical absorption spectrum in optical density units (O.D) of the LiF crystal (LiFC2) irradiated with $8 \mathrm{keV}$ X-rays on a uniform area. The formation of stable primary $\mathrm{F}$ centers and aggregate defects was inferred by the presence of the main $\mathrm{F}$ and $\mathrm{M}$ absorption bands. Figure $1 \mathrm{~b}$ shows the PLE spectra of the sample LiFC2 acquired at two different emission wavelengths (541 nm and $678 \mathrm{~nm}$ ) corresponding to $\mathrm{F}_{3}{ }^{+}$and $\mathrm{F}_{2} \mathrm{CC}$, respectively. The PLE spectra show the excitation wavelength for which the PL emission of $\mathrm{F}_{2}$ and $\mathrm{F}_{3}{ }^{+}$defects is optimized. The results demonstrate that for both CCs a blue light is preferred for efficiently exciting their PL emission in the red and green spectral ranges. In this regard, Figure 1c reports the PL spectrum of the LiFC2 sample acquired at the excitation wavelength of $445 \mathrm{~nm}$, which consists in the overlapping of the two broad emission bands in the green and red spectral intervals due to $\mathrm{F}_{3}{ }^{+}$and $\mathrm{F}_{2} \mathrm{CCs}$, respectively.

(a)

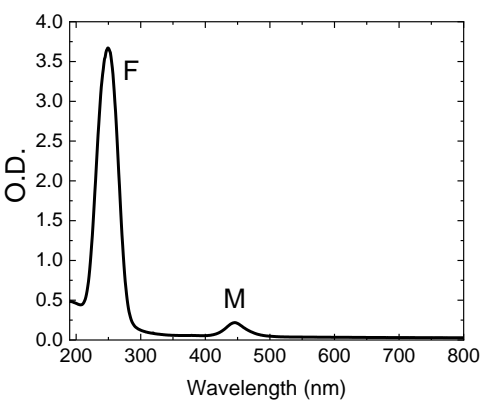

(b)

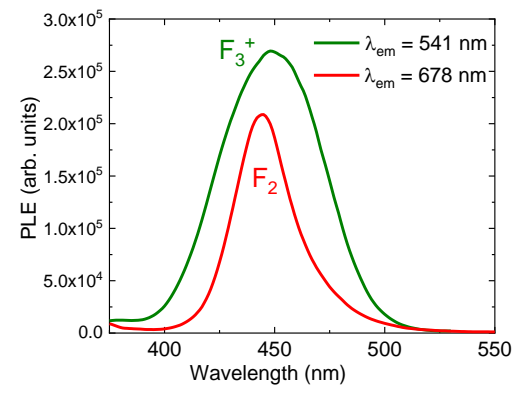

(c)

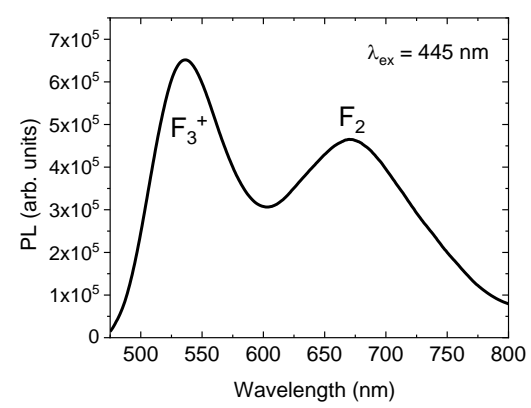

Figure 1. (a) Room temperature absorption spectrum in optical density units (O.D); (b) PLE spectra and (c) PL spectrum of the LiF crystal irradiated with the $8 \mathrm{keV}$ X-ray beam (LiFC2).

Figure 2 shows the fluorescence images (overlapping $\mathrm{F}_{3}{ }^{+}$and $\mathrm{F}_{2} \mathrm{CC}$ signals) of the same test pattern stored in the LiFC1 sample irradiated with the broadband white beam at different exposure times and detected with the confocal fluorescence microscope. For each circular spot, the position was obtained by moving the LiF crystal in the X and Y directions perpendicularly to the beam axis and the irradiation was performed at different $X$-ray exposure times. This picture, obtained by using a low-magnification objective of the fluorescence microscope, demonstrates that LiF detectors are able to store X-ray images in a large field of view with high spatial resolution, as also reported in Figure 3. The high dynamic range of the detector is demonstrated by its capability to store images with very different exposure times and it is limited by the dynamic range of the reading system 
(12 bit). In a previous paper [7], the absorption and PL intensities of $\mathrm{F}_{3}{ }^{+}$and $\mathrm{F}_{2} \mathrm{CCs}$ as functions of irradiation times were investigated and the linear behavior of both signals was observed.

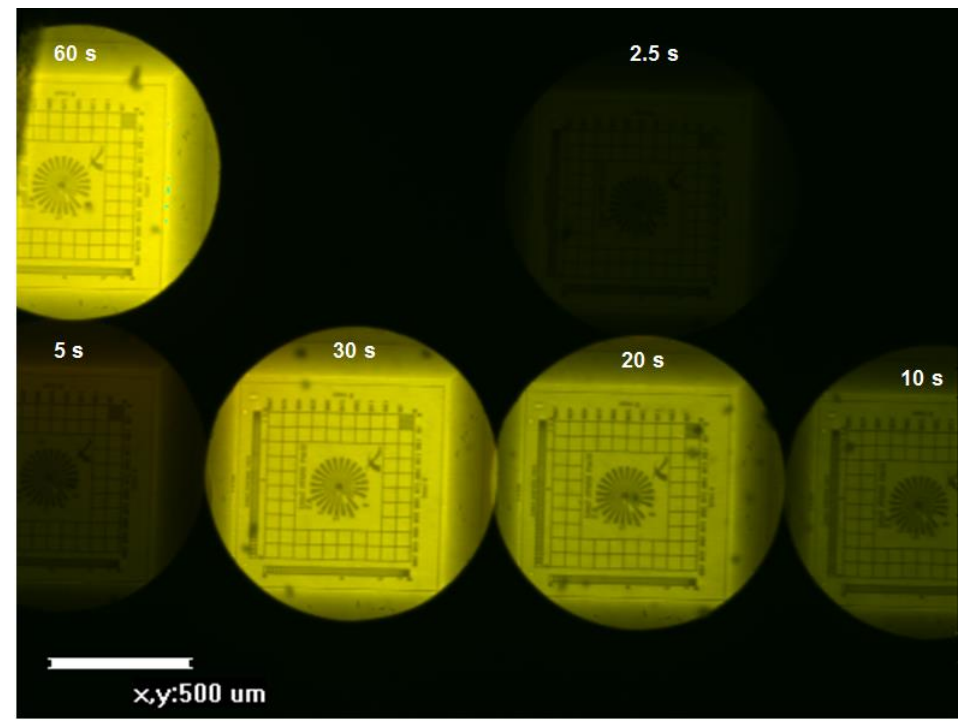

Figure 2. Fluorescence images of test pattern stored in the LiFC1 sample irradiated with the broadband white beam. Each circular spot with the test pattern image, whose position was obtained by moving LiF crystal in the $\mathrm{X}$ and $\mathrm{Y}$ directions perpendicularly to the beam axis, was irradiated with X-ray exposure times.

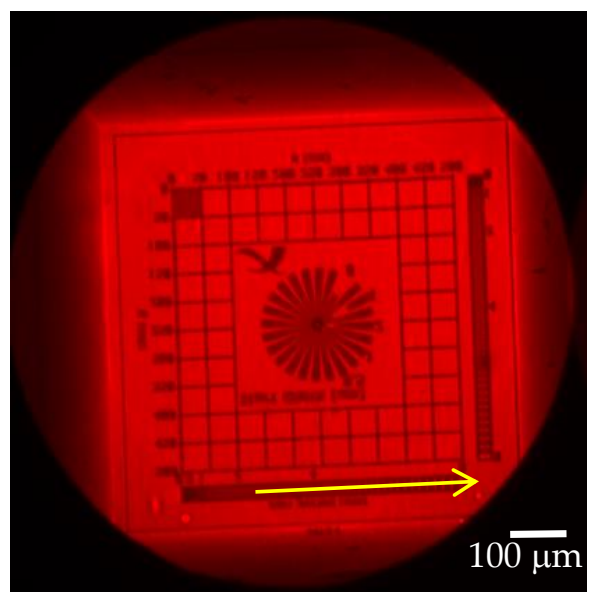

(a)

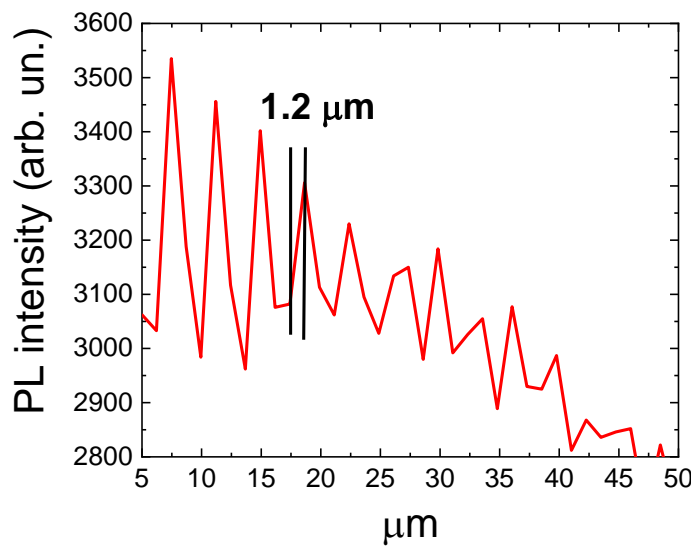

(b)

Figure 3. (a) 2D fluorescent image acquired by the CLSM of the irradiated LiF crystal LiFC1: image of the test pattern obtained by irradiating with the white broadband X-ray beam for $30 \mathrm{~s}$ irradiation time. (b) Intensity profile of the PL signal along a pattern in a) with decreasing spatial period (yellow arrow).

Figure 3a shows the red $\left(\mathrm{F}_{2}\right)$ photoluminescent 2D image acquired by the CLSM of the irradiated LiF crystal LiFC1 storing the projected image of the test pattern obtained by irradiating for $30 \mathrm{~s}$ with the white broadband X-ray beam. Figure $3 \mathrm{~b}$ reports the intensity profile of the PL signal along a periodic structure of the test pattern with decreasing period; it may be observed that the micrometric details of the pattern $X$-ray image stored in the $\mathrm{LiF}$ crystal are well resolved.

By using the optical sectioning operation of the CLSM system along the optical $\mathrm{Z}$ axis by controlled spatial increments, several slices along the colored volume of the irradiated $500-\mu \mathrm{m}$ thick LiF crystal were obtained. A set of red ( $\left.F_{2} \mathrm{CCs}\right) 2 \mathrm{D}$ images at different 
$\mathrm{Z}$ positions detected in fluorescence mode are shown in Figure 4a. The software of the confocal microscope, after acquisition of the 2D slices, performs a 3D reconstruction of the images (Figure $4 \mathrm{~b}$ ) and it can show $\mathrm{Z}$ sections along $\mathrm{X}, \mathrm{Y}$ directions of the acquired volume as reported in Figure 4c-e. Results in Figure 4 clearly show that the X-ray image of the pattern is well resolved along the thickness of the irradiated LiF crystal.

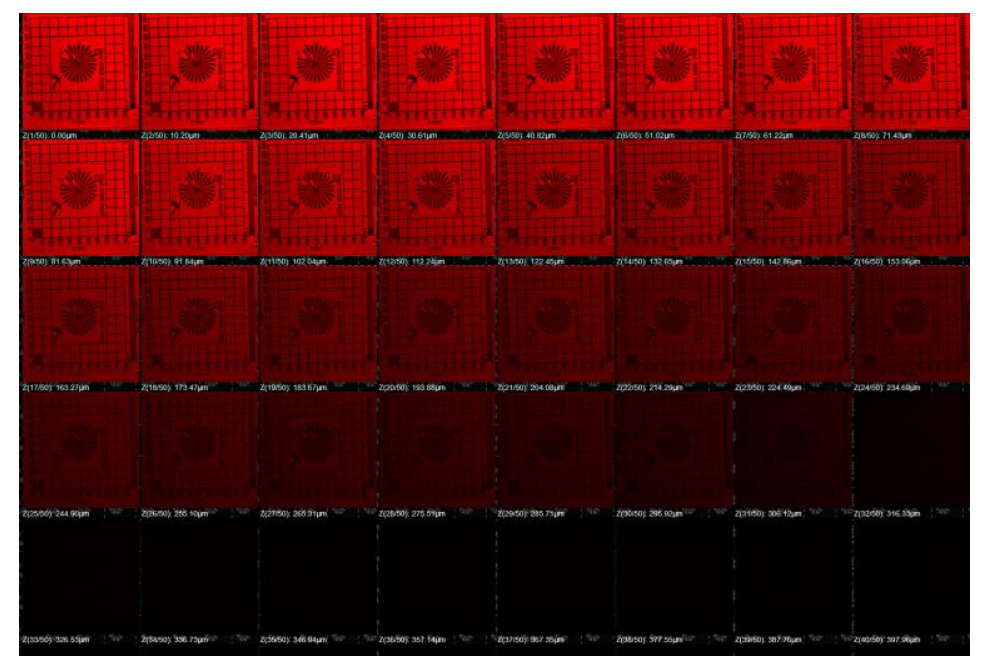

(a)

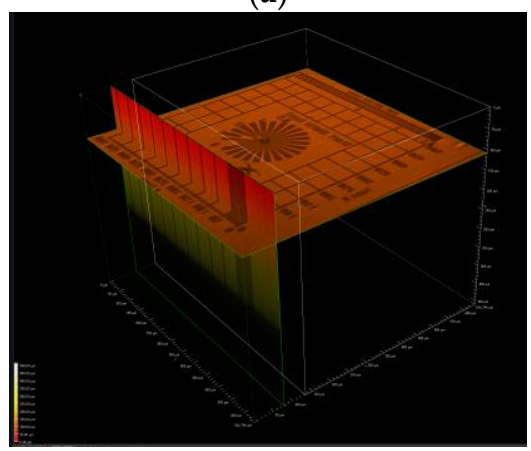

(c)

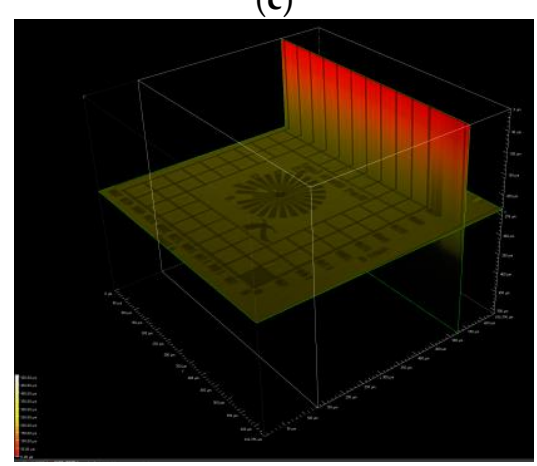

(e)

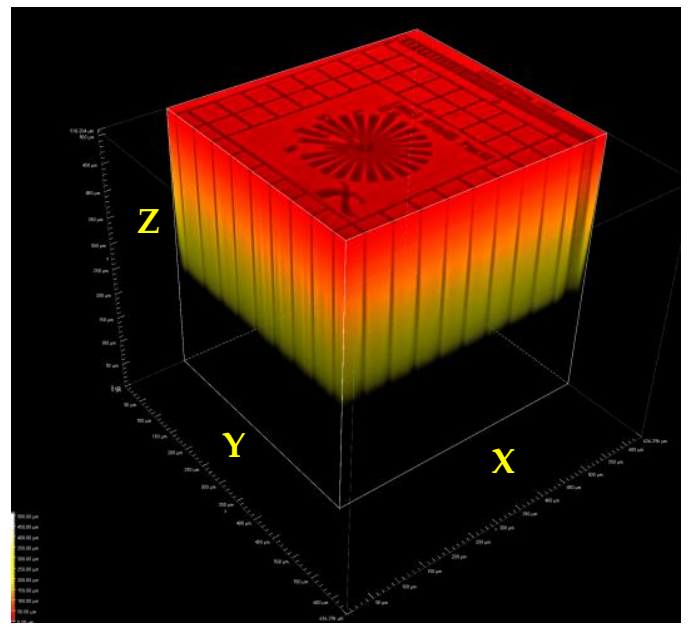

(b)

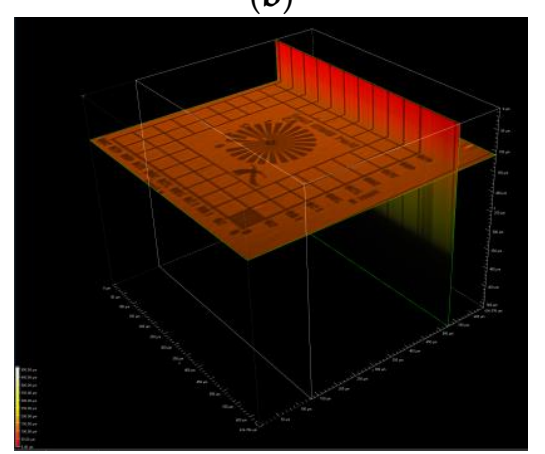

(d)

Figure 4. (a) Set of $2 \mathrm{D}\left(X Y: 636 \times 636 \mu \mathrm{m}^{2}\right)$ slices for different $Z$ positions $(Z$ interval $=0-500 \mu \mathrm{m} ; Z$ step $=10 \mu \mathrm{m})$ of the photoluminescent colored volume of the LiF crystal irradiated with a broadband X-ray beam (LiFC1 exposure time $30 \mathrm{~s})$; (b) $3 D$ reconstruction $\left(X Y=636 \times 636 \mu \mathrm{m}^{2}, Z=500 \mu \mathrm{m}\right)$ of $(\mathbf{a})$. Color scale $(0-500) \mu \mathrm{m} ;(\mathbf{c}-\mathbf{e}) X Y$ slices at different $Z$ positions together with $X Z$ slices at different $Y$ positions of the acquired colored volume. Color scale (0-500) $\mu \mathrm{m}$.

Figure 5 a reports the $3 \mathrm{D}$ reconstruction $\left(X Y=636 \times 636 \mu \mathrm{m}^{2}, \mathrm{Z}=1 \mathrm{~mm}\right)$ of the colored volume obtained by detecting the $\mathrm{F}_{2}$ PL signal of the $8 \mathrm{keV} X$-ray irradiated LiF crystal on a uniform area (sample LiFC2) by means of the CLSM. A selected XZ slice of the $3 \mathrm{D}$ reconstruction showing the $\mathrm{Z}$ distribution of the $\mathrm{F}_{2}$ fluorescence intensity along the thickness of the LiF crystal $(1 \mathrm{~mm})$ is shown in Figure $5 \mathrm{~b}$. Figure $5 \mathrm{c}$ reports the PL 
intensity profile along the $\mathrm{Z}$ scan optical axis of the 3D image reported in Figure $5 \mathrm{~b}$. According to the $X$-ray transmission properties in solids [15], the energy deposition of the X-ray beam exponentially decreases along the penetration depth in LiF. Assuming that $\mathrm{CC}$ concentrations are proportional to the deposited energy, the confocal fluorescence microscope is an appropriate technique to investigate the $\mathrm{X}$-ray colored profile along the crystal thickness ( $\mathrm{Z}$ direction). The experimental $\mathrm{F}_{2}$ PL profile (red curve) is shown in Figure $5 \mathrm{c}$ together with its best fit with a single exponential curve (blue curve), a value of $(236+/-3) \mu \mathrm{m}$, corresponding to the $1 / \mathrm{e}$ of the maximum, being obtained. The theoretical $\mathrm{X}$-ray attenuation length in $\mathrm{LiF}$ corresponding to an X-ray energy of $\sim 8 \mathrm{keV}$ is $331 \mu \mathrm{m} \mathrm{[15].}$ Since possible re-absorption phenomena of pumping laser by CCs or reabsorption of the CC PL during CLSM Z-scanning measurements could occur in this 3D characterization, a comparison with the confocal Raman micro-spectrometer was performed.

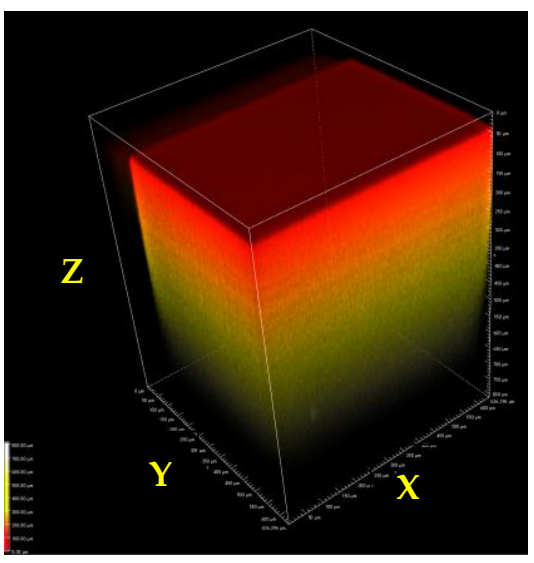

(a)

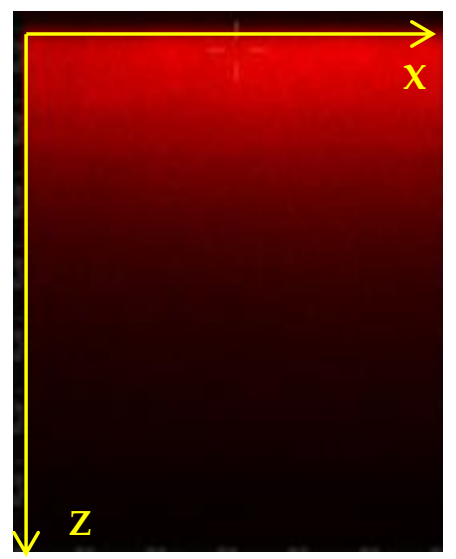

(b)

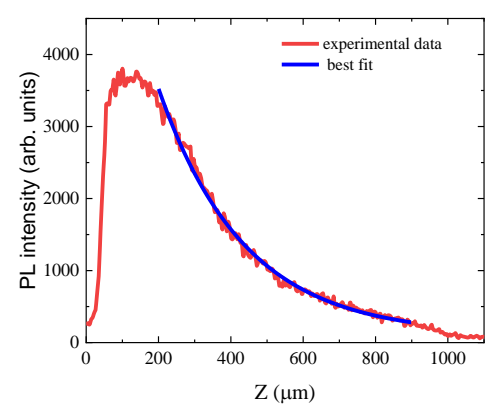

(c)

Figure 5. (a) $3 \mathrm{D}$ reconstruction $\left(X Y=636 \times 636 \mu \mathrm{m}^{2}, Z=1 \mathrm{~mm}\right)$ of the photoluminescent volume acquired by CLSM of the $8 \mathrm{keV}$ X-ray irradiated LiF crystal (sample LiFC2) on a uniform area. Color scale (0-800) $\mu \mathrm{m}(\mathbf{b}) \mathrm{A}$ XZ slice of the (a) showing the $\mathrm{Z}$ distribution of $\mathrm{F}_{2} \mathrm{CC}$ fluorescence intensity along the thickness of the LiF crystal (1 mm); (c) PL intensity profile along $Z$ scan optical axis of $(\mathbf{b})$ and its best fit with a single exponential curve.

The results of the comparison between the Raman spectrum acquired with a 532-nm excitation wavelength in a point of a colored area of the LiF crystal (LiFC1) irradiated by the white beam (exposure time $30 \mathrm{~s}$ ) and the one acquired in a point of a not-colored region are shown in Figure 6a. It is evident that the presence of aggregated color centers produces the appearance of the first order peak of the LiF Raman spectrum. Figure $6 \mathrm{~b}$ reports a 2D Raman map of an area of the LiFC1 with two colored spots obtained with X-ray exposure times of $30 \mathrm{~s}$ and $20 \mathrm{~s}$. The Raman map is obtained by scanning the sample in a prefixed grid and using the band peak intensity at about $220 \mathrm{~cm}^{-1}$ as contrasting parameter. In Figure $6 \mathrm{~b}$, a higher Raman signal for the spot with the higher irradiation time is evident. By using the optical sectioning of the confocal Raman spectrometer, several 2D Raman maps along the colored volume of the irradiated $500-\mu \mathrm{m}$ thick LiF crystal were acquired. A 3D Raman map (Figure $6 \mathrm{c}$ ) of a selected region $\left(X Y=400 \times 800 \mu \mathrm{m}^{2}, Z=0-500 \mu \mathrm{m}, \mathrm{Z}\right.$ step $=30 \mu \mathrm{m}$ ) of the spot irradiated for $60 \mathrm{~s}$ (highlighted in the yellow rectangle on the fluorescence image in the inset on the left) has been obtained (nominal excitation laser power of $75 \mathrm{~mW}$ ). Figure $6 \mathrm{~d}$ reports XY, XZ, YZ slices of the Figure 6c. Figure 6e reports 2D (XY) Raman map slices for $Z=0$ (top surface), $Z=-250 \mu \mathrm{m}$ and $Z=-500 \mu \mathrm{m}$ (bottom surface). 


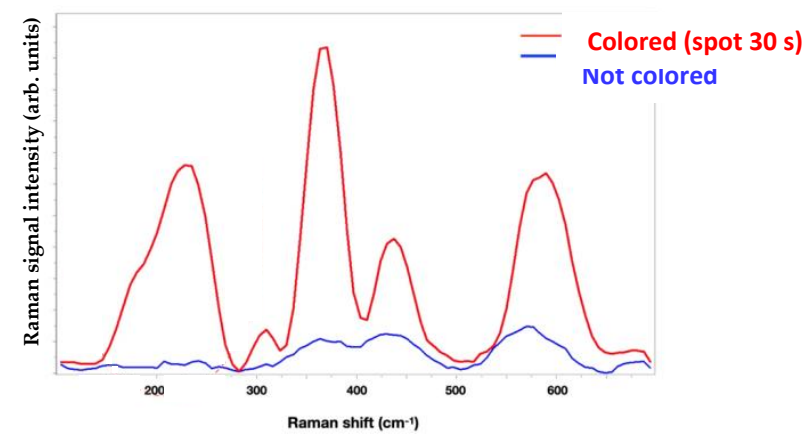

(a)

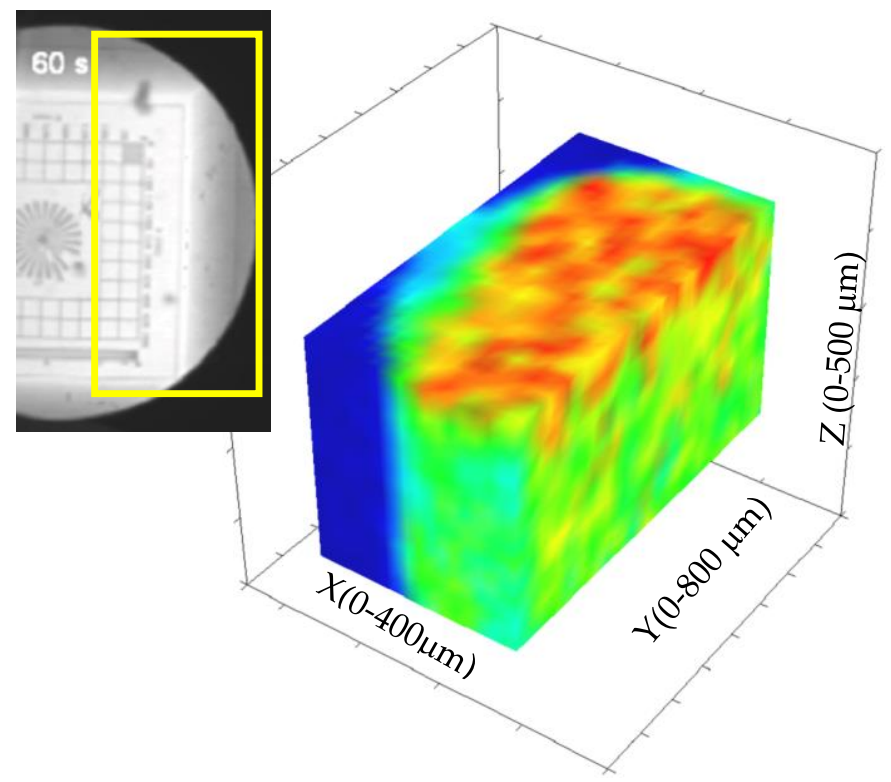

(c)
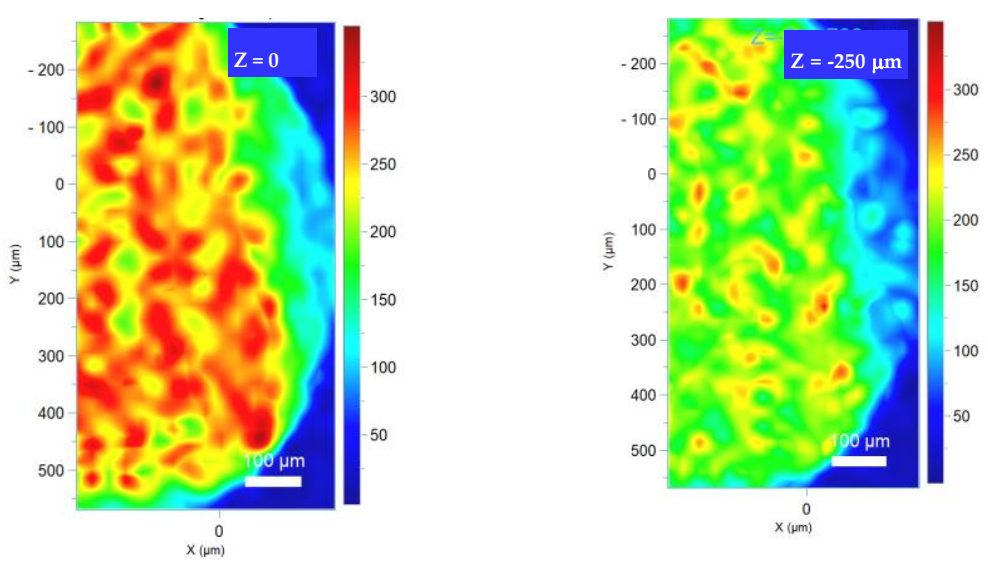

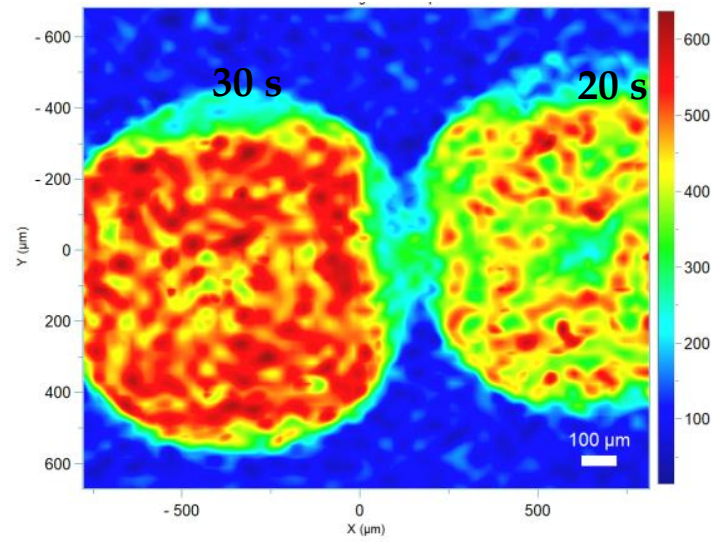

(b)
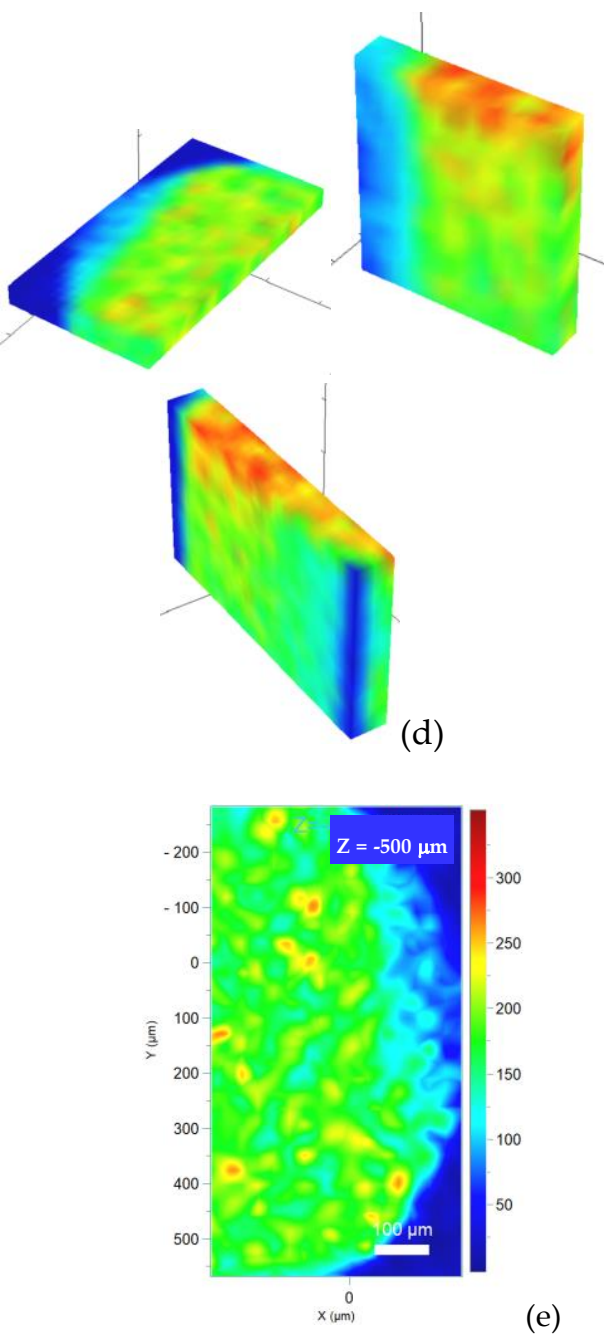

Figure 6. (a) Raman spectra acquired in a point of a colored area of the LiF crystal (LiFC1) irradiated with broadband X-rays (exposure time $30 \mathrm{~s}$ ) and in a not-colored region; (b) 2D Raman map of an area of the irradiated LiF crystal LiFC1 with two colored spots obtained with X-ray exposure times of $30 \mathrm{~s}$ and $20 \mathrm{~s}$; (c) 3D Raman maps $\left(X Y=400 \times 800 \mu \mathrm{m}^{2}\right.$, $Z=0-500 \mu \mathrm{m}$, Zstep $=30 \mu \mathrm{m}$ ) of the spot irradiated for $60 \mathrm{~s}$; the inset on the left: reports the corresponding 2D fluorescence image acquired with confocal fluorescence microscope where the region selected for Raman map has been highlighted with a yellow rectangle; (d) XY, XZ, YZ slices of (c); (e) 2D Raman map slices along the colored volume of the irradiated 500- $\mu \mathrm{m}$ thick $\mathrm{LiF}$ crystal $Z=0$ (top surface), $Z=-250 \mu \mathrm{m}$ and $Z=-500 \mu \mathrm{m}$ (bottom surface). Color scale: Raman signal intensity (0-350) arb. units. 
Figure 7a reports the 3D Raman maps $\left(X Y=182 \times 172 \mu \mathrm{m}^{2}, \mathrm{Z}=0-1 \mathrm{~mm}\right)$ of the uniform colored area of the $1000-\mu \mathrm{m}$ thick LiF crystal (LiFC2) irradiated with $8 \mathrm{keV}$ X-rays and obtained by using the optical sectioning of the confocal Raman spectrometer along the optical $\mathrm{Z}$ axis. An XZ slice of the Raman map is reported in Figure $7 \mathrm{~b}$ for a region containing irradiated and not irradiated areas. The absence of the Raman signal for the not-colored region is evident. The Raman intensity profile along the $Z$ direction (yellow arrow) for the colored region (magenta curve) is reported in Figure $7 \mathrm{c}$ together with its best fit with a single exponential curve (blue curve). A value of $(221+/-3) \mu \mathrm{m}$ corresponding to the $1 / \mathrm{e}$ of the maximum, was obtained. This value is close to that obtained with red fluorescence signal in the CLSM systems (see Figure 5c). Further investigations regarding optical sectioning of the confocal systems applied to X-ray colored LiF crystals are in progress.

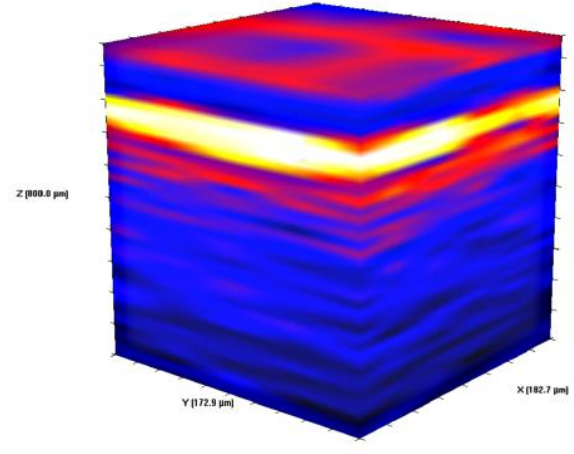

(a)

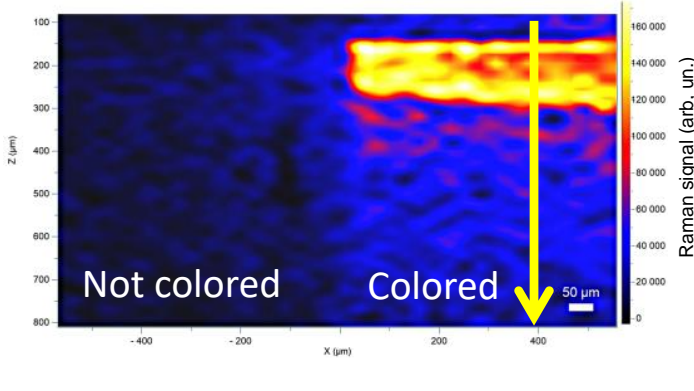

(b)

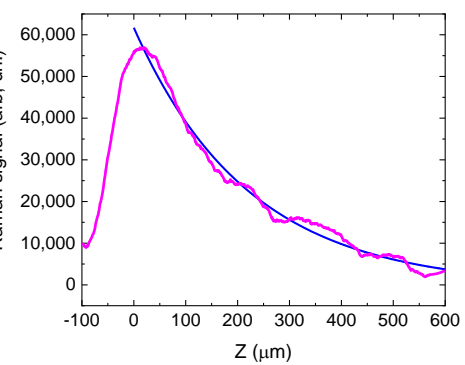

(c)

Figure 7. (a) 3D Raman maps $\left(X Y=182 \times 172 \mu \mathrm{m}^{2}, Z=0-1 \mathrm{~mm}\right)$ of the uniform colored area of the 1-mm thick LiF crystal (LiFC2) irradiated with $8 \mathrm{keV}$ X-rays. (b) XZ slice of the Raman map for a region containing irradiated and not irradiated area. Color scale: Raman signal intensity $(0-160,000)$ arb. units; (c) Raman intensity profile along Z direction for the colored region together with its best fit.

\section{Conclusions}

LiF crystals irradiated with the broadband white $X$-ray beam and with monochromatic $8 \mathrm{keV}$ X-rays at KIT synchrotron light source (Karlsruhe, Germany) have been investigated with confocal optical systems operating in fluorescence microscopy and Raman microspectroscopy modes; 3D fluorescence and Raman maps of colored LiF crystals have been performed, obtaining volumetric reconstructions of the X-ray-induced CC distributions. The combination of the fluorescence maps with the Raman ones could represent a suitable method for investigations of X-irradiated LiF crystals with confocal techniques as advanced tool for 3D X-ray detection.

Author Contributions: Conceptualization, F.B. and S.B.; methodology, F.B., S.B., A.C., E.N., V.N., M.P. and M.A.V.; formal analysis, E.N. and F.B.; investigation, F.B., S.B., A.C., E.N., V.N., M.P. and M.A.V.; writing-original draft preparation, F.B.; writing-review and editing, S.B., A.C., R.M.M., E.N., V.N., M.P. and M.A.V.; project administration, R.M.M.; funding acquisition, R.M.M. All authors have read and agreed to the published version of the manuscript.

Funding: Part of this research has been carried out within the TECHEA (Technologies for Health) Project, funded by the Italian National Agency for New Technologies, Energy and Sustainable Economic Development (ENEA), Italy.

Conflicts of Interest: The authors declare no conflict of interest.

\section{References}

1. Nahum, J.; Wiegand, D.A. Optical Properties of SomeF-Aggregate Centers in LiF. Phys. Rev. 1967, 154, 817-830. [CrossRef]

2. Ustione, A.; Cricenti, A.; Bonfigli, F.; Flora, F.; Lai, A.; Marolo, T.; Montereali, R.M.; Baldacchini, G.; Faenov, A.; Pikuz, T.; et al. Scanning near-field optical microscopy images of microradiographs stored in lithium fluoride films with an optical resolution of त/12. Appl. Phys. Lett. 2006, 88, 141107. [CrossRef] 
3. Almaviva, S.; Bonfigli, F.; Franzini, I.; Lai, A.; Montereali, R.M.; Pelliccia, D.; Cedola, A.; Lagomarsino, S. Hard x-ray contact microscopy with $250 \mathrm{~nm}$ spatial resolution using a LiF film detector and table-top microsource. Appl. Phys. Lett. 2006, 89, 054102. [CrossRef]

4. Bonfigli, F.; Hartley, N.; Inubushi, Y.; Koenig, M.; Matsuoka, T.; Makarov, S.; Montereali, R.M.; Nichelatti, E.; Ozaki, N.; Piccinini, M.; et al. Photoluminescence properties and characterization of LiF-based imaging detector irradiated by $10 \mathrm{keV} \mathrm{XFEL} \mathrm{beam.} \mathrm{Opt.}$ Damage Mater. Process. By EUV/X-Ray Radiat. VII 2019, 11035, 110350N. [CrossRef]

5. Baldacchini, G.; Bollanti, S.; Bonfigli, F.; Flora, F.; di Lazzaro, P.; Lai, A.; Marolo, T.; Montereali, R.M.; Murra, D.; Faenov, A.; et al. A novel soft X-ray submicron imaging detector based on point defects in LiF. Rev. Sci. Instrum. 2005, 76, 113104-1-12. [CrossRef]

6. Bonfigli, D.H.F.; Dabagov, S.B.; Montereali, R.M.; Della Ventura, G.; Bellatreccia, F.; Magi, M. LiFdetectors-polycapillary lens for advanced X-ray imaging. Nucl. Instrum. Methods Phys. Res. A 2013, 720, 113-115.

7. Bonfigli, F.; Cecilia, A.; Bateni, S.H.; Nichelatti, E.; Pelliccia, D.; Somma, F.; Vagovic, P.; Vincenti, M.; Baumbach, T.; Montereali, R. In-line X-ray lensless imaging with lithium fluoride film detectors. Radiat. Meas. 2013, 56, 277-280. [CrossRef]

8. Bonfigli, F.; Faenov, A.; Flora, F.; Francucci, M.; Gaudio, P.; Lai, A.; Martellucci, S.; Montereali, R.M.; Pikuz, T.; Reale, L.; et al. High-resolution water window X-ray imaging of in vivo cells and their products using LiF crystal detectors. Microsc. Res. Tech. 2008, 71, 35-41. [CrossRef] [PubMed]

9. Gasilov, S.; Faenov, A.; Pikuz, T.; Skobelev, I.; Boldarev, A.; Gasilov, V.; Magunov, A.; Fukuda, Y.; Kando, M.; Kotaki, H.; et al. Nanostructure Conventional And Propagation-based Phase Contrast Imaging Using Femtosecond Laser Cluster Plasma Source and LiF Crystal Soft Xray Detectors. Contrib. Plasma Phys. 2009, 49, 488-495. [CrossRef]

10. Imaging detectors based on photoluminescence of radiation-induced defects in lithium fluoride for XFEL beam monitoring. Il Nuovo Cim. 2020, 42, 1-8.

11. Pikuz, T.; Faenov, A.; Matsuoka, T.; Matsuyama, S.; Yamauchi, K.; Ozaki, N.; Albertazzi, B.; Inubushi, Y.; Yabashi, M.; Tono, K.; et al. 3D visualization of XFEL beam focusing properties using LiF crystal X-ray detector. Sci. Rep. 2015, 5, 17713. [CrossRef] [PubMed]

12. Kurobori, T.; Takemura, A.; Miyamoto, Y.; Maki, D.; Koguchi, Y.; Takeuchi, N.; Yamamoto, T.; Chen, Y.Q. A disk-type dose imaging detector based on blue and orange RPL in Ag-activated phosphate glass for 2D and 3D dose imaging applications. Radiat. Meas. 2015, 83, 51-55. [CrossRef]

13. Diaspro, A. Confocal and Two-Photon Microscopy. Foundations, Applications and Advances; Diaspro, A., Ed.; Wiley-Liss: New York, NY, USA, 2002.

14. Bateni, S.H.; Bonfigli, F.; Cecilia, A.; Baumbach, T.; Pelliccia, D.; Somma, F.; Vincenti, M.; Montereali, R. Optical characterisation of lithium fluoride detectors for broadband X-ray imaging. Nucl. Instruments Methods Phys. Res. Sect. A Accel. Spectrometers Detect. Assoc. Equip. 2012, 720, 109-112. [CrossRef]

15. X-Ray Interactions with Matter. Available online: http://henke.lbl.gov/optical_constants/ (accessed on 15 September 2021). 